\title{
How to compare market efficiency? The Sharpe ratio based on the ARMA-GARCH forecast
}

Lin Liu* (D) and Qiguang Chen

* Correspondence: liulin@pku.edu.cn Peking University, Beijing 100871, China

\begin{abstract}
This paper derives a new method for comparing the weak-form efficiency of markets. The author derives the formula of the Sharpe ratio from the ARMA-GARCH model and finds that the Sharpe ratio just depends on the coefficients of the AR and MA terms and is not affected by the GARCH process. For empirical purposes, the Sharpe ratio can be formulated with a monotonic increasing function of $R$-squared if the sample size is large enough. One can utilize the Sharpe ratio to compare weak-form efficiency among different markets. The results of stochastic simulation demonstrate the validity of the proposed method. The author also constructs empirical AR-GARCH models and computes the Sharpe ratio for S\&P 500 Index and the SSE Composite Index.

Keywords: ARMA, GARCH, Measurement of market efficiency, Sharpe ratio, Stochastic simulation

Mathematics subject classification: $60 \mathrm{G} 10,62 \mathrm{M} 10$

JEL classification: G10, G14, G17, C22
\end{abstract}

\section{Introduction}

Since Fama (1970) published his influential paper, there has been a thorough assessment of market efficiency. Various methods have been proposed over the decades to test the efficient market hypothesis (EMH), but the empirical results vary according to the specific markets chosen, periods of time, and even the selected methods. For instance, like the frictionless state in physics, the efficient market could just be viewed as an ideal state with no real existence in the world. However, it could serve as a useful benchmark for measuring relative efficiency of markets over space and time.

In fact, it is more relevant to measure the relative efficiency of markets from the empirical perspective. Just as Campbell et al. (1997) pointed out, "The notion of relative efficiency-the efficiency of one market measured against another-may be a more useful concept than the all-or-nothing view taken by much of the traditional marketefficiency literature."

(c) The Author(s). 2020 Open Access This article is licensed under a Creative Commons Attribution 4.0 International License, which permits use, sharing, adaptation, distribution and reproduction in any medium or format, as long as you give appropriate credit to the original author(s) and the source, provide a link to the Creative Commons licence, and indicate if changes were made. The images or other third party material in this article are included in the article's Creative Commons licence, unless indicated otherwise in a credit line to the material. If material is not included in the article's Creative Commons licence and your intended use is not permitted by statutory regulation or exceeds the permitted use, you will need to obtain permission directly from the copyright holder. To view a copy of this licence, visit http://creativecommons.org/licenses/by/4.0/. 
So far, researchers have tended to measure and investigate market efficiency using various statistical methods. One market efficiency measure is based on autocorrelation of returns. The idea behind this method is that a strong autocorrelation reflects a deviation from random walk pricing and is indicative of violations of the EMH. For example, the Q-statistic of Box and Pierce (1970) is usually used as a measure for market efficiency. Lo and Mackinlay (1988) proposed a test statistic, the ratio of variances, which is also used as a measure for market efficiency. Another type of market efficiency measure reflects the degree to which returns are associated with past market returns; it is similar to the delay measure of Mech (1993) and Hou and Moskowitz (2005). The logic behind it is that a security price has a lower efficiency if it incorporates the information contained in the market index movement slower than another.

The literature mentioned above is based on the EMH, which uses many assumptions to describe the model, such as independence, limited variance, normality, and linear paradigm, among many others. However, some literature that tests the market efficiency relies on an alternative theory known as the fractal market hypothesis (FMH). This theory does not have the aforementioned strict assumptions but instead focuses on the nonlinearities in the financial market and supposes that the behavior of investors is affected by their level of understanding of the information along with the investment period. Based on the FMH, Liu et al. (1999) used a Hurst exponent and a time-varying Hurst exponent to describe the dynamic changes of market efficiency in the Hong Kong REITS market. They determined that the market had not yet reached the state of weak efficiency. Han et al. (2019) compared the efficiency of five primary indexes of different boards of the Chinese stock market using multifractal detrended fluctuation analysis (MF-DFA). This method measures the multifractal degrees. The authors found that the SHSE-SZS300 index that reflects the overall trend of the Shanghai and Shenzhen main board markets was less efficient than the ChiNext index, which had the lowest multifractal lever among five indexes. Moradi et al. (2019) used the L-Co-R algorithm analyze the fractal features of the Tehran Stock Exchange and the London Stock Exchange. In essence, fractal theory is a type of chaos theory to some extent. Ola et al. (2014) applied the local polynomial approximation model, which is a method relating to chaos processes and testing to determine the efficiency of the Tehran Stock Exchange and found that the efficiency of the market could not be confirmed. Moreover, as the efficiency of the market is related to investor behavior, some works have indirectly analyzed the efficiency of the market from this perspective, for example, Wen et al. (2019).

In principle, profitability is at the core of market efficiency. According to Malkiel (1992), "Moreover, efficiency with respect to an information set ... implies that it is impossible to make economic profits by trading on the basis of [that information set]." However, the traditional measures of market efficiency do not reflect profitability. For example, using data from 56 markets, Griffin et al. (2010) found that variance ratios and market delay measures often showed greater deviation from random walk pricing in developed markets than emerging markets. However, they also found that shortterm reversal strategies and momentum strategies could earn similar returns in both types of markets. Therefore, they argued that the usual efficiency measures might yield misleading inferences. 
It is regarded that a proper measure of market efficiency should directly reflect the expected return and the relevant risk. The Sharpe ratio (SR) is one of the main criteria of portfolio assessment, and it has been widely used to evaluate the performance of mutual funds. Sharpe (1966) proposed the now well-known Sharpe ratio to evaluate a fund's return in excess of the risk-free rate, adjusted by the additional risk the fund assumes. A high Sharpe ratio indicates superior risk-adjusted performance.

The Sharpe ratio can help measure market efficiency. Fortunately, one can derive the formula of the Sharpe ratio from the ARMA-GARCH model. Financial returns are often modeled as autoregressive time series with random disturbances having conditional heteroscedastic variances, especially with GARCH type processes that have been extensively used in the financial and econometric literature as risk models of many financial time series.

The relationship between the SR and the $R^{2}$ of the ARMA-GARCH model is investigated in this paper. This is related to the research on return predictability by the $R^{2}$ of the regression of stock return on predictors implied by the rational asset pricing model. These research studies compare the observed $R^{2}$ of predictive regressions with the maximal bounds permitted by asset pricing models, and through the comparison, they conclude whether the market is effective. Kirby (1998) first proposed the frame of analyzing the restrictions on predictability implied by the rational asset pricing model. Based on similar frameworks, Levich and Potì (2015) constructed an upper bound on the explanatory power of predictive regressions motivated by "no good-deal" restrictions in the currency market and found that the currency predictability exceeded the bound, thereby showing that the market is not effective. Huang and Zhou (2017) provided two theoretical upper bounds on the $R^{2}$ of the regression and reported that the predictive $R^{2}$ was significantly larger than the upper bounds. Their bounds were tighter than Ross (2005), who is a pioneer of providing the bounds on predictability for all asset pricing models under no arbitrage conditions. Potì (2018) proposed an upper bound on the predictability of asset returns, which was at least as tight as Huang and Zhou (2017).

However, we do not make a comparison between the predicted $R^{2}$ and the theoretical boundaries based on asset pricing models under capital market equilibrium because we do not measure the degree of market efficiency precisely but only compare the market efficiency among markets. So, we focus on testing whether the SR can show differences in market efficiency. We find that the SR can be formulated with a monotonic increasing function of $R^{2}$. We also compare the SR with another indicator proposed by Zhang (1999), namely conditional entropy.

This paper proceeds as follows. In "The Sharpe Ratio based on the ARMA-GARCH Forecast" section, the formula of the SR is derived from the ARMA-GARCH model. "Stochastic simulation" section applies the method through stochastic simulation and provides a comparison with the conditional entropy indicator. "Empirical Research" section presents the empirical results of the S\&P 500 Index and the SSE Composite Index. "Conclusion" section gives concluding remarks.

\section{The Sharpe ratio based on the ARMA-GARCH forecast}

To compare technical analysis with the time series forecast, Fang and $\mathrm{Xu}$ (2003) derived the expected value of a one-period excess return from the AR (1) model forecast. 
Following on these methods, we derive the expected value of a one-period excess return from the general $\operatorname{ARMA}(p, q)-\operatorname{GARCH}(r, s)$ model forecast. Furthermore, we deduce its standard deviation so that the formula of the SR can be obtained.

\section{The basic approach}

We start with the ARMA $(p, q)$ model.

Let $S_{t}, t=0,1, \cdots, T$ denote stock prices observed at discrete moments. Let $Y_{t}$ denote the continuously compounded return at time $t$. So:

$$
Y_{t}=\ln \left(\frac{S_{t}}{S_{t-1}}\right), \quad t=1,2, \cdots, T
$$

Suppose $\left\{Y_{t}\right\}$ satisfies the following $\operatorname{ARMA}(p, q)$ process:

$$
Y_{t}=\sum_{i=1}^{p} a_{i} Y_{t-i}+\sum_{i=1}^{q} b_{i} \varepsilon_{t-i}+\varepsilon_{t}
$$

where $\left\{\varepsilon_{t}\right\}$ is the Gaussian white noise, $W N\left(0, \sigma^{2}\right)$. Neither $a_{p}$ nor $b_{q}$ is equal to zero.

Provided that all the roots of

$$
1-\sum_{i=1}^{p} a_{i} z^{i}=0
$$

lie outside the unit circle, it is straightforward to verify that $\left\{Y_{t}\right\}$ is a covariancestationary process.

According to eq. (2.2), we can express $Y_{t+1}$ as

$$
Y_{t+1}=\sum_{i=1}^{p} a_{i} Y_{t-i+1}+\sum_{i=1}^{q} b_{i} \varepsilon_{t-i+1}+\varepsilon_{t+1} .
$$

Let $I_{t}$ denote the information set at time $t$, which includes the present and past records of both $\left\{\varepsilon_{t}\right\}$ and $\left\{Y_{t}\right\}$. Then the forecast of $Y_{t+1}$ based on $I_{t}$ is

$$
E_{t}\left[Y_{t+1}\right]=E\left[Y_{t+1} \mid I_{t}\right]=\sum_{i=1}^{p} a_{i} Y_{t-i+1}+\sum_{i=1}^{q} b_{i} \varepsilon_{t-i+1} .
$$

Now, eq. (2.3) can be simplified to

$$
Y_{t+1}=E_{t}\left[Y_{t+1}\right]+\varepsilon_{t+1}
$$

As is known, $Y_{t+1}$ in eq. (2.3) can also be formulated with an $\mathrm{MA}(\infty)$ process:

$$
Y_{t+1}=\sum_{i=0}^{\infty} \psi_{i} \varepsilon_{t-i+1} .
$$

Because $\left\{\varepsilon_{t}\right\}$ is the Gaussian white noise, both $Y_{t+1}$ and $E_{t}\left[Y_{t+1}\right]$ follow the Gaussian distribution with zero mean.

For simplicity, we assume that the risk-free return and the trading cost rate are both zero. Then, it is trivial that the strategy at time $t$ that maximizes the expected value of the one-period excess return can be formulated as follows.

Definition 1: A buy (sell) signal is generated at time $t$ if 


$$
E_{t}\left[Y_{t+1}\right]>0(<0) \text {. }
$$

Accordingly, the expected value of the one-period excess return is

$$
\begin{aligned}
\mu_{g}= & \operatorname{Prob}\left(E_{t}\left(Y_{t+1}\right)>0\right) \cdot E\left\{Y_{t+1} \mid E_{t}\left[Y_{t+1}\right]>0\right\} \\
& -\operatorname{Prob}\left(E_{t}\left(Y_{t+1}\right)<0\right) \cdot E\left\{Y_{t+1} \mid E_{t}\left[Y_{t+1}\right]<0\right\} \\
= & E\left\{Y_{t+1} \mid E_{t}\left[Y_{t+1}\right]>0\right\} .
\end{aligned}
$$

If the standard deviation of $E_{t}\left[Y_{t+1}\right]$ is known and denoted by $\sigma_{E}$, it's easy to verify (proof in Appendix) that

$$
\mu_{g}=\frac{\sigma_{E} \sqrt{2}}{\sqrt{\pi}}
$$

Because $\varepsilon_{t+1}$ and $E_{t}\left[Y_{t+1}\right]$ are uncorrelated, we can compute $\sigma_{E}$ according to the variance of $Y_{t+1}$, which is denoted by $\gamma_{0}$. So,

$$
\sigma_{E}=\sqrt{\gamma_{0}-\sigma^{2}}
$$

According to the strategy given by Definition 1, we can verify that the standard deviation of the one-period excess return is (proof in Appendix)

$$
\sigma_{g}=\left[\sigma^{2}+\left(1-\frac{2}{\pi}\right) \sigma_{E}^{2}\right]^{\frac{1}{2}}
$$

Therefore, the Sharpe ratio is

$$
S R=\frac{\mu_{g}}{\sigma_{g}}=\frac{\sigma_{E} \sqrt{2}}{\sqrt{\pi \sigma^{2}+(\pi-2) \sigma_{E}^{2}}}=\left[\frac{\pi}{2}\left(1+\frac{\sigma^{2}}{\sigma_{E}^{2}}\right)-1\right]^{-\frac{1}{2}},
$$

or

$$
S R=\sqrt{\frac{2\left(\gamma_{0}-\sigma^{2}\right)}{2 \sigma^{2}+(\pi-2) \gamma_{0}}} .
$$

According to formula (2.12) or (2.13), we conclude that the Sharpe ratio lies in a specific scope:

$$
S R \in\left[0, \sqrt{\frac{2}{\pi-2}}\right) .
$$

In fact, $\gamma_{0}$ is a function of $\sigma^{2}$. For a few simple processes such as the AR (1), AR (2), $\operatorname{MA}(q)$, and ARMA $(1,1)$, we are able to derive the formula of $\gamma_{0}$ and thereby formulate the SR with the parameters of the original model.

For a general $\operatorname{ARMA}(p, q)$ process, it is difficult to obtain the formula of $\gamma_{0}$, but we can adopt the following ways to calculate its value.

First, according to Box et al. (2008), the series $\left\{\psi_{j}\right\}$ in eq. (2.6) can be computed recursively from the following relations:

$$
\psi_{j}=\left\{\begin{array}{cl}
1, & j=0 \\
b_{j}+\sum_{k=1}^{p} a_{k} \psi_{j-k}, & j=1,2, \cdots,
\end{array}\right.
$$

with $\psi_{j}=0$ for $j<0$, and $b_{j}=0$ for $j>q$. 
Because the values of $\left\{\psi_{j}\right\}$ decay geometrically toward zero, we need to compute only a few terms.

Secondly, it is easy to calculate the value of $\gamma_{0}$ according to eq. (2.6).

$$
\gamma_{0}=\sigma^{2} \sum_{j=0}^{\infty} \psi_{j}^{2}
$$

From eq. (2.13), (2.14), and (2.15), we find that the SR just depends on the autoregressive coefficients, $a_{1}, \cdots, a_{p}$, and the moving average coefficients, $b_{1}, \cdots, b_{q}$.

For empirical purposes, we can estimate the SR by means of formula (2.13), in which $\sigma$ can be replaced by the standard error of the regression, and $\gamma_{0}$ can be estimated as the sample variance of $\left\{Y_{t}\right\}$. In fact, if the sample size is large enough, formula (2.13) can be reduced to

$$
S R \approx \sqrt{\frac{2 R^{2}}{\pi-2 R^{2}}}
$$

Formula (2.16) shows the SR is a monotonic increasing function of the $R$-squared. Therefore, the $R$-squared in time series models is of great significance with respect to market efficiency. The R-squared in time series models can be used as a measure of market efficiency.

The relationship between the SR and R-squared can also be shown as the curve in Fig. 1.

From Fig. 1, we see that the relationship between the SR and $R$-squared is approximately linear positive dependence when the value of $R$-squared is larger than 0.1.

Cochrane (1999) derived a similar result from the following model:

$$
Y_{t+1}=E(Y)+b\left[X_{t}-E(X)\right]+\varepsilon_{t+1} .
$$

The author demonstrated that the SR associated with a market timing strategy based on this predictor equation is given by

$$
S R=\sqrt{\frac{S R_{0}^{2}+R^{2}}{1-R^{2}}},
$$

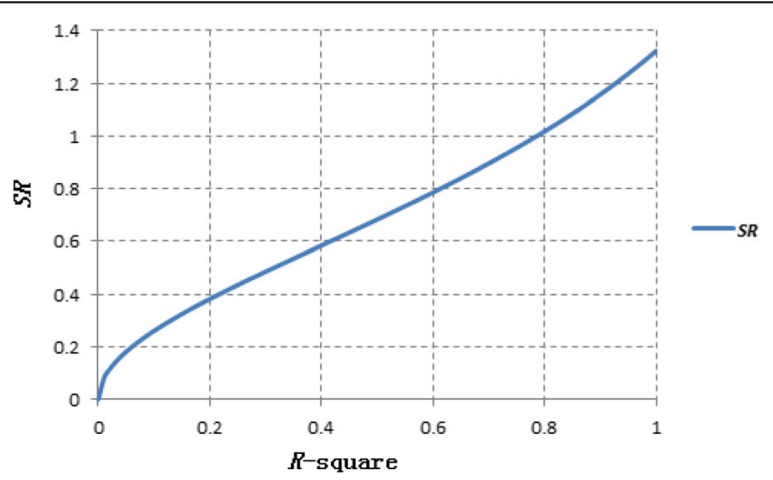

Fig. 1 The relationship between the Sharpe ratio and R-squared. The horizontal axis shows the R-squared, and the vertical axis shows the Sharpe ratio 
where $S R_{0}$ is the SR based on a buy-and-hold strategy, and $R$-squared is taken from the predictor equation.

It is obvious that the SR in formula (2.16') is also a monotonic increasing function of $R$-squared. Nevertheless, if $\left\{Y_{t}\right\}$ satisfies the $\operatorname{ARMA}(p, q)$ process, it is improper to use formula (2.16'), which gives an overestimate of the SR.

\section{Several simple examples}

\section{The AR (1) process}

To start with, we consider the AR (1) process:

$$
Y_{t}=a_{1} Y_{t-1}+\varepsilon_{t}
$$

where $0<\left|a_{1}\right|<1$.

It is easy to verify

$$
\begin{aligned}
& \gamma_{0}=\frac{\sigma^{2}}{1-a_{1}^{2}}, \\
& \sigma_{E}=\frac{\left|a_{1}\right| \sigma}{\sqrt{1-a_{1}^{2}}} .
\end{aligned}
$$

First, substituting eq. (2.19) into eq. (2.9) gives

$$
\mu_{g}=\frac{\left|a_{1}\right| \sigma \sqrt{2}}{\sqrt{\pi\left(1-a_{1}^{2}\right)}} .
$$

Then, substituting eq. (2.19) into eq. (2.11) gives

$$
\sigma_{g}=\sigma\left[\frac{\pi-2 a_{1}^{2}}{\pi\left(1-a_{1}^{2}\right)}\right]^{\frac{1}{2}}
$$

Finally, substituting eq. (2.19) into eq. (2.12) gives

$$
S R=\frac{\sqrt{2}\left|a_{1}\right|}{\sqrt{\pi-2 a_{1}^{2}}} .
$$

Now it can be seen that the SR just depends on $a_{1}$ and is not affected by the variance of $\varepsilon_{t}$. The smaller the absolute value of $a_{1}$ is, the lower the SR is. The SR is equal to zero when $a_{1}$ is equal to zero.

\section{The AR (2) process}

In order to derive the formula of $\gamma_{0}$ for the AR (2) or ARMA $(1,1)$ processes, we make use of the autocovariance-generating function. According to Hamilton (1994), the autocovariance-generating function for a stationary $\operatorname{ARMA}(p, q)$ process can be written as:

$$
g_{y}(z)=\frac{\sigma^{2}\left(1+b_{1} z+b_{2} z^{2}+\cdots\right)\left(1+b_{1} z^{-1}+b_{2} z^{-2}+\cdots\right)}{\left(1-a_{1} z-a_{2} z^{2}-\cdots\right)\left(1-a_{1} z^{-1}-a_{2} z^{-2}-\cdots\right)} .
$$

If we convert expression (2.23) to a polynomial of $z$, then the coefficient of $z^{0}$ is exactly $\gamma_{0}$.

Now, we consider the AR (2) process: 


$$
Y_{t}=a_{1} Y_{t-1}+a_{2} Y_{t-2}+\varepsilon_{t},
$$

where $0<\left|a_{2}\right|<1, a_{2} \pm a_{1}<1$.

By the way proposed above, we obtain

$$
\gamma_{0}=\frac{\left(1-a_{2}\right) \sigma^{2}}{\left(1+a_{2}\right)\left[\left(1-a_{2}\right)^{2}-a_{1}^{2}\right]} .
$$

Substituting formula (2.25) into formula (2.10) gives

$$
\sigma_{E}=\sigma *\left\{\frac{1-a_{2}}{\left(1+a_{2}\right)\left[\left(1-a_{2}\right)^{2}-a_{1}^{2}\right]}-1\right\}^{\frac{1}{2}} .
$$

Then, substituting formula (2.26) into formula (2.9) gives

$$
\mu_{g}=\frac{\sigma \sqrt{2}}{\sqrt{\pi}} * \sqrt{\frac{1-a_{2}}{\left(1+a_{2}\right)\left[\left(1-a_{2}\right)^{2}-a_{1}^{2}\right]}-1 .}
$$

Now, substituting formula (2.26) into formula (2.11) gives

$$
\sigma_{g}=\sigma\left[\frac{\pi\left(1-a_{2}\right)-2\left(a_{1}^{2}+a_{2}^{2}\right)+2 a_{2}\left(a_{2}^{2}-a_{1}^{2}\right)}{\pi\left(1+a_{2}\right)\left[\left(1-a_{2}\right)^{2}-a_{1}^{2}\right]}\right]^{\frac{1}{2}} .
$$

In the end, dividing eq. (2.27) by eq. (2.28) gives

$$
S R=\frac{\mu_{g}}{\sigma_{g}}=\left[\frac{2\left[\left(a_{1}^{2}+a_{2}^{2}\right)+a_{2}\left(a_{1}^{2}-a_{2}^{2}\right)\right]}{\pi\left(1-a_{2}\right)-2\left[\left(a_{1}^{2}+a_{2}^{2}\right)+a_{2}\left(a_{1}^{2}-a_{2}^{2}\right)\right]}\right]^{\frac{1}{2}} .
$$

Now it can be seen that the SR just depends on $a_{1}$ and $a_{2}$, and it is not affected by the variance of $\varepsilon_{t}$. From formula (2.29), we can see that the AR (2) process is reduced to the AR (1) process when $a_{2}$ is equal to zero. Furthermore, both $\mu_{g}$ and SR will be zero if both $a_{1}$ and $a_{2}$ are equal to zero.

\section{The ARMA $(1,1)$ process}

Now, let us consider the ARMA $(1,1)$ process. In a similar way, we obtain

$$
\begin{aligned}
& \gamma_{0}=\frac{\left(1+2 a_{1} b_{1}+b_{1}^{2}\right) \sigma^{2}}{1-a_{1}^{2}}, \\
& \sigma_{E}=\frac{\left|a_{1}+b_{1}\right| \sigma}{\sqrt{1-a_{1}^{2}}}, \\
& \mu_{g}=\frac{\left|a_{1}+b_{1}\right| \sigma \sqrt{2}}{\sqrt{\pi *\left(1-a_{1}^{2}\right)}}, \\
& \sigma_{g}=\sigma\left[1+\left(1-\frac{2}{\pi}\right) \frac{\left(a_{1}+b_{1}\right)^{2}}{1-a_{1}^{2}}\right]^{\frac{1}{2}}, \\
& S R=\frac{\sqrt{2}\left|a_{1}+b_{1}\right|}{\sqrt{\pi\left(1+2 a_{1} b_{1}+b_{1}^{2}\right)-2\left(a_{1}+b_{1}\right)^{2}}} .
\end{aligned}
$$


Now, it can be seen that the SR just depends on $a_{1}$ and $b_{1}$, and it is not affected by the variance of $\varepsilon_{t}$.

It is obvious that the SR will be zero only if

$$
a_{1}+b_{1}=0 .
$$

In fact, at this point, the ARMA $(1,1)$ process is equivalent to white noise, so it is impossible to realize any positive return.

In addition, it is easy to verify that formula (2.34) is equivalent to formula (2.22) only if

$$
b_{1}=-2 a_{1} .
$$

Then, the SR is the same for the ARMA $(1,1)$ and AR (1) processes.

The SR for the ARMA $(1,1)$ process is approximately equal to that for the AR (1) process if both $a_{1}$ and $b_{1}$ are small enough (e.g., less than 0.1) and the coefficient $a^{\prime}{ }_{1}$ in the AR (1) meets the following condition:

$$
\left|a^{\prime}\right|=\left|a_{1}+b_{1}\right| .
$$

\section{The MA (2) process}

Finally, we consider the MA (2) process and compare the SR with the Q-statistic of Box and Pierce (1970).

For the MA (2) process, it is easy to verify

$$
\begin{aligned}
& \gamma_{0}=\left(1+b_{1}^{2}+b_{2}^{2}\right) \sigma^{2}, \\
& \gamma_{1}=\left(b_{1}+b_{1} b_{2}\right) \sigma^{2}, \\
& \gamma_{2}=b_{2} \sigma^{2}, \\
& \gamma_{j}=0, \text { for } j>2 .
\end{aligned}
$$

Substituting the expression of $\gamma_{0}$ into formula (2.13) gives

$$
S R=\left[\frac{2\left(b_{1}^{2}+b_{2}^{2}\right)}{\pi+(\pi-2)\left(b_{1}^{2}+b_{2}^{2}\right)}\right]^{\frac{1}{2}} .
$$

Now, it can be seen that the SR just depends on $b_{1}$ and $b_{2}$, and it is not affected by the variance of $\varepsilon_{t}$.

The autocorrelation parameters of $\left\{Y_{t}\right\}$ are as follows:

$$
\begin{aligned}
& \rho_{1}=\frac{\gamma_{1}}{\gamma_{0}}=\frac{b_{1}+b_{1} b_{2}}{1+b_{1}^{2}+b_{2}^{2}}, \\
& \rho_{2}=\frac{\gamma_{2}}{\gamma_{0}}=\frac{b_{2}}{1+b_{1}^{2}+b_{2}^{2}}, \\
& \rho_{j}=0, \text { for } j>2 .
\end{aligned}
$$

According to Box and Pierce (1970), the Q-statistic at lag $k$ is a test statistic for the null hypothesis that there is no autocorrelation up to order $k$. It is computed as: 


$$
Q=T(T+2) \sum_{j=1}^{k} \frac{r_{j}^{2}}{T-j}
$$

where $r_{j}$ is the $j$-th sample's autocorrelation and $T$ is the number of observations.

For large $T, r_{j}$ approximates $\rho_{j}$, so expression (2.36) can be reduced to

$$
Q \approx T \sum_{j=1}^{k} \rho_{j}^{2}
$$

Formula (2.37) shows that the $Q$-statistic is proportional to the sample size, $T$. Therefore, it is unreasonable to use the $Q$-statistic as a measure of market efficiency if the sample size is ignored.

Applying formula (2.37) to the MA (2) process gives

$$
Q=\frac{T\left[b_{1}^{2}\left(1+b_{2}\right)^{2}+b_{2}^{2}\right]}{1+b_{1}^{2}+b_{2}^{2}}
$$

or

$$
Q / T=\frac{b_{1}^{2}\left(1+b_{2}\right)^{2}+b_{2}^{2}}{1+b_{1}^{2}+b_{2}^{2}} .
$$

Now, we consider whether the ratio of $Q$ to $T$ can be used to measure market efficiency. For example, given $b_{1}=0.5$, let us change the value of $b_{2}$ from -0.45 to 0.45 and observe how the values of $S R$ and $Q / T$ change with it (Fig. 2).

Figure 2 shows that the Sharpe ratio achieves the minimum when $b_{2}$ is equal to zero and the $S R$ curve is symmetrical. In contrast, $Q / T$ achieves the minimum when $b_{2}$ is

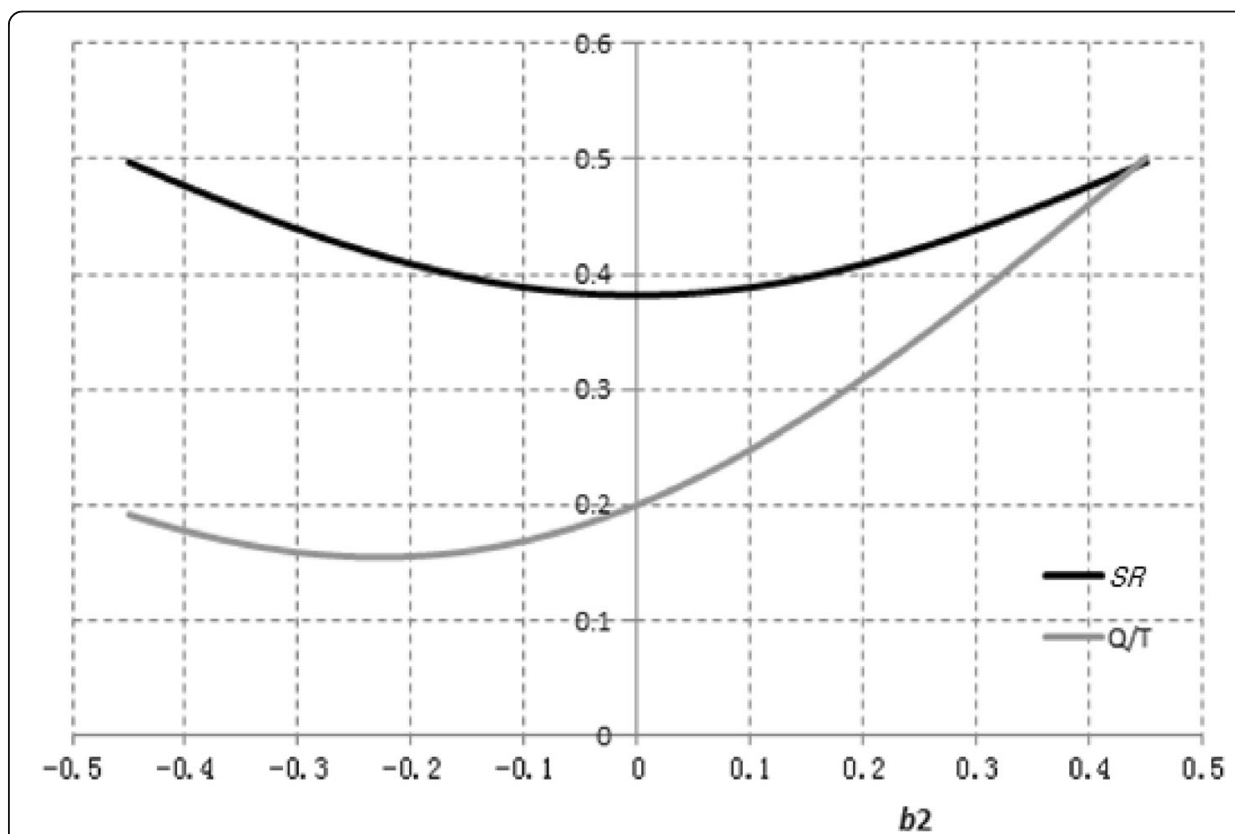

Fig. 2 Comparison of the Sharpe ratio and $Q / T$ for the MA (2) Process. The horizontal axis shows $b_{2}$, the coefficient of the second moving average term. The vertical axis shows the Sharpe ratio or the ratio of the Q-statistic to sample size 
equal to -0.23 , and the curve is asymmetrical. It is obvious that ranking by $Q / T$ is different from ranking by $S R$. Therefore $Q / T$ is not a proper measure of market efficiency.

\section{The ARMA-GARCH model}

Now, we consider the general ARMA-GARCH model.

If the $\left\{\varepsilon_{t}\right\}$ in expression (2.2) follows a $\operatorname{GARCH}(r, s)$ process:

$$
\begin{aligned}
\varepsilon_{t} & =\sqrt{h_{t}} \cdot v_{t}, \\
h_{t} & =\phi+\sum_{j=1}^{r} \alpha_{j} h_{t-j}+\sum_{k=1}^{s} \beta_{k} \varepsilon_{t-k}^{2},
\end{aligned}
$$

where $\left\{v_{t}\right\}$ is the Gaussian white noise, $\operatorname{WN}\left(0, \sigma^{2}\right)$, and $\phi>0, \alpha_{j}>0, j=1, \cdots, r, \beta_{k}>0$, $k=1, \cdots, s, \sum_{j=1}^{r} \alpha_{j}+\sum_{k=1}^{s} \beta_{k}<1$.

According to $\mathrm{Lu}(1999)$, we can conclude that $\left\{\varepsilon_{t}\right\}$ is a covariance-stationary process:

$$
\begin{aligned}
& E\left(\varepsilon_{t}\right)=0, \\
& \operatorname{Var}\left(\varepsilon_{t}\right)=\frac{\phi}{1-\sum_{j=1}^{r} \alpha_{j}-\sum_{k=1}^{s} \beta_{k}}, \\
& \operatorname{Cov}\left(\varepsilon_{j}, \varepsilon_{k}\right)=0, j \neq k .
\end{aligned}
$$

Because $v_{t+1}$ and $E_{t}\left[Y_{t+1}\right]$ are uncorrelated, $\varepsilon_{t+1}$ and $E_{t}\left[Y_{t+1}\right]$ are uncorrelated. So, we can compute the standard deviation of $E_{t}\left[Y_{t+1}\right]$ according to formula (2.10) if we use $\sqrt{\frac{\phi}{1-\sum_{j=1}^{r} \alpha_{j}-\sum_{k=1}^{s} \beta_{k}}}$ to replace $\sigma$. In fact, all results are the same as the ARMA model if $\sigma$ is replaced by

$$
\sqrt{\frac{\phi}{1-\sum_{j=1}^{r} \alpha_{j}-\sum_{k=1}^{s} \beta_{k}}}
$$

Furthermore, the SR is not affected by the variance of $\varepsilon_{t}$, so it is not affected by the GARCH process. The SR just depends on the coefficients of the $\operatorname{ARMA}(p, q)$ process, namely $a_{1}, \cdots, a_{p}, b_{1}, \cdots, b_{q}$.

\section{Stochastic simulation}

We explore the proposed method by means of stochastic simulation. We study the AR (1), ARMA $(1,1)$, and AR $(1)$-GARCH $(1,1)$ processes, which serve as three examples.

\section{Stochastic simulation of the AR (1) process}

\section{Simulation about risk}

Given a set of parameters, according to eq. (2.17), we simulated the AR (1) process to produce a series consisting of 10,000 observations. Then, we simulated trading in accordance with the strategy described in Definition 1 and generated a series of returns whose standard deviation (denoted by $\sigma_{\text {simul }}$ ) can be computed and compared with the value from formula (2.21).

The results for nine sets of parameters are displayed in Table 1. 
Table 1 Results on risk for the AR (1) process. The table reports the actual standard deviations of the simulated returns and their theoretical values for nine AR (1) processes with different parameters. The actual standard deviation of the simulated returns is denoted by $\sigma_{\text {simul, }}$ and its theoretical value is denoted by $\sigma_{q}$

\begin{tabular}{llllllllll}
\hline Group & $(1)$ & $(2)$ & $(3)$ & $(4)$ & $(5)$ & $(6)$ & $(7)$ & $(8)$ & $(9)$ \\
\hline$a_{1}$ & 0.1 & 0.4 & 0.8 & 0.1 & 0.4 & 0.8 & 0.1 & 0.4 & 0.8 \\
$\sigma$ & 0.01 & 0.01 & 0.01 & 0.02 & 0.02 & 0.02 & 0.03 & 0.03 & 0.03 \\
$\sigma_{g}$ & 0.0100 & 0.0103 & 0.0128 & 0.0200 & 0.0207 & 0.0257 & 0.0301 & 0.0310 & 0.0385 \\
$\sigma_{\text {simul }}$ & 0.0101 & 0.0102 & 0.0128 & 0.0203 & 0.0206 & 0.0255 & 0.0301 & 0.0314 & 0.0381 \\
\hline
\end{tabular}

From Table 1, we can see the values of $\sigma_{\text {simul }}$ are very close to the values of $\sigma_{g}$. The results confirm formula (2.21).

\section{Simulation about return}

Now, we specify the parameters as follows:

$$
a_{1}=0.1, \sigma=0.01 \text {. }
$$

Substituting them into formula (2.20) gives

$$
\mu_{g}=0.0802 \%
$$

Similarly, we simulated to produce a series consisting of 1000 observations according to eq. (2.17). Then, we simulated trading according to the strategy described in Definition 1 and generated a series of returns whose average value (denoted by $R$ ) can be computed and compared with the value from formula (2.20).

We carried out the simulation 100 times and found the mean of $R$ to be $0.0793 \%$, which approximates $0.0802 \%$.

Figure 3 shows the distribution of $R$ along with its summary statistics.

It can be seen that $R$ approximately follows the Gaussian distribution.

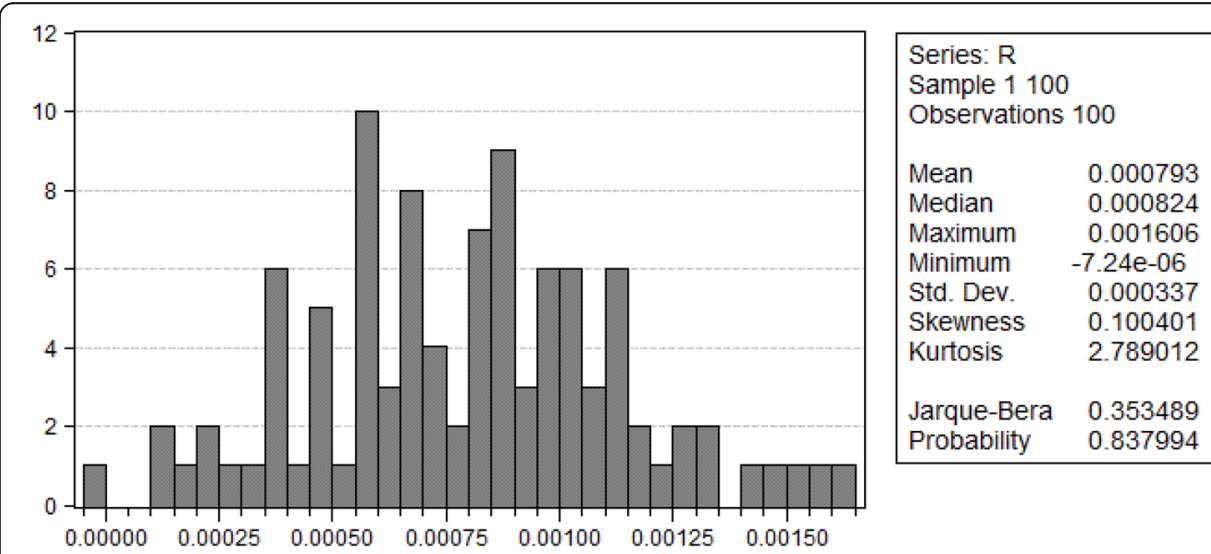

Fig. 3 The distribution and summary statistics of the average simulated return for the AR (1) process. We use $R$ to denote the average value of the simulated returns. The distribution of $R$ is shown in the left, and the summary statistics are listed in the right 
Table 2 Results on risk for the ARMA $(1,1)$ process. The table reports the actual standard deviations of the simulated returns and their theoretical values for nine ARMA $(1,1)$ processes with different parameters. The actual standard deviation of the simulated returns is denoted by $\sigma_{\text {simul, }}$ and its theoretical value is denoted by $\sigma_{g}$

\begin{tabular}{lllllllll}
\hline Group & $(1)$ & $(2)$ & $(3)$ & $(4)$ & $(5)$ & $(6)$ & $(7)$ & $(8)$ \\
\hline$a_{1}$ & 0.5 & 0.5 & -0.5 & -0.5 & 0.5 & 0.5 & -0.5 & -0.5 \\
$b_{1}$ & 0.3 & -0.3 & 0.3 & -0.3 & 0.3 & -0.3 & 0.3 & -0.3 \\
$\sigma$ & 0.01 & 0.01 & 0.01 & 0.01 & 0.03 & 0.03 & 0.03 & 0.03 \\
$\sigma_{g}$ & 0.0114 & 0.0101 & 0.0101 & 0.0114 & 0.0343 & 0.0303 & 0.0303 & 0.0343 \\
$\sigma_{\text {simul }}$ & 0.0114 & 0.0103 & 0.0101 & 0.0115 & 0.0346 & 0.0301 & 0.0301 & 0.0344 \\
\hline
\end{tabular}

\section{Stochastic simulation of the ARMA $(1,1)$ process}

\section{Simulation about risk}

First, given a set of parameters, we simulated to produce a series consisting of 10, 000 observations according to eq. (2.3). Then, we simulated trading according to the strategy described in Definition 1 and generated a series of returns whose standard deviation (denoted by $\sigma_{\text {simul }}$ ) can be computed and compared with the value from formula (2.33).

The results for eight sets of parameters are displayed in Table 2.

Just like Tables 1 and 2 shows that the value of $\sigma_{\text {simul }}$ is very close to the value of $\sigma_{g}$. The results confirm formula (2.33).

\section{Simulation about return}

Now, we specify the parameters as follows:

$$
a_{1}=0.075, b_{1}=-0.03, \sigma=0.015
$$

According to formula (2.32), we obtain

$$
\mu_{g}=0.0540 \%
$$

We simulated to produce a series consisting of 1000 observations according to eq. (2.3). Then, we simulated trading according to the strategy described in Definition 1

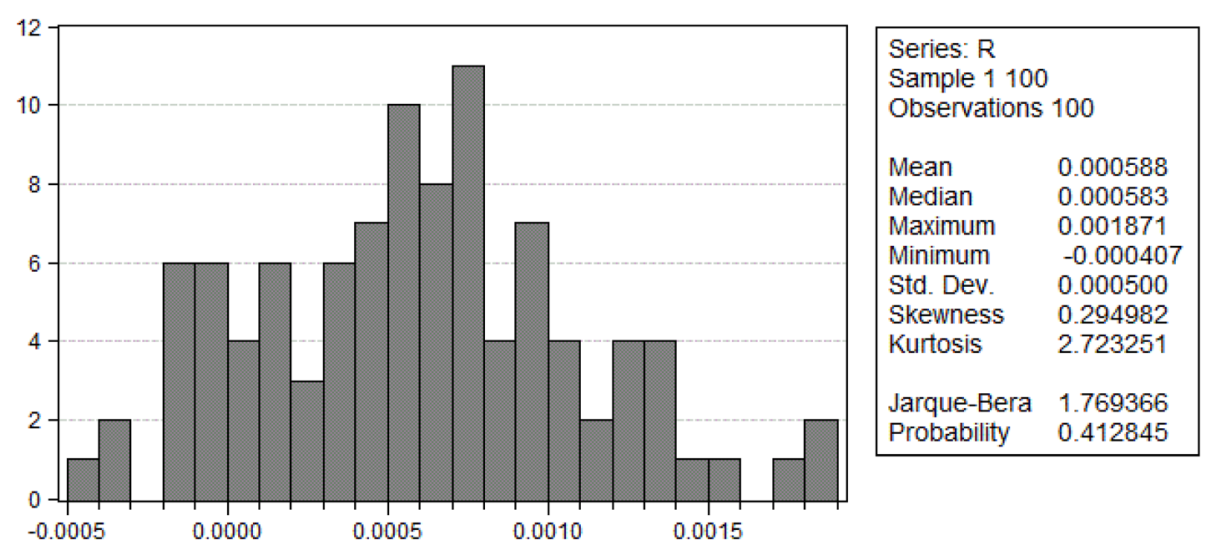

Fig. 4 The distribution and summary statistics of the average simulated return for the ARMA $(1,1)$ process. We use $R$ to denote the average value of the simulated returns. The distribution of $R$ is shown in the left, and the summary statistics are listed in the right 
and generate a series of returns whose average value (denoted by $R$ ) can be computed and compared with the value from formula (2.32).

We carried out the simulation 100 times and found the mean of $R$ to be $0.0588 \%$, which approximates $0.0540 \%$. In fact, their difference can be accounted for by the low value of the SR, which was only 0.036 .

Figure 4 shows the distribution of $R$ along with its summary statistics.

It can be seen that $R$ approximately follows the Gaussian distribution.

\section{Stochastic simulation of the AR (1)-GARCH $(1,1)$ process}

\section{Simulation about risk}

Given a set of parameters, according to eqs. (2.17), (2.40), and (2.41), we simulated the AR (1)-GARCH $(1,1)$ process to produce a series consisting of 10,000 observations. Then, we simulated trading in accordance with the strategy described in Definition 1 and generated a series of returns whose standard deviation (denoted by $\sigma_{\text {simul }}$ ) can be computed and compared with the value from the following formula:

$$
\sigma_{g}=\sqrt{\frac{\phi\left(\pi-2 a_{1}^{2}\right)}{\pi\left(1-a_{1}^{2}\right)\left(1-\alpha_{1}-\beta_{1}\right)}} .
$$

The results for eight sets of parameters are displayed in Table 3.

From Table 3, we can see the values of $\sigma_{\text {simul }}$ are very close to the values of $\sigma_{g}$. The results confirm formula (3.1).

\section{Simulation about return}

Now, the expected value of the one-period excess return is

$$
\mu_{g}=\sqrt{\frac{2 \phi a_{1}^{2}}{\pi\left(1-a_{1}^{2}\right)\left(1-\alpha_{1}-\beta_{1}\right)}} .
$$

We specify the parameters as follows:

$$
a_{1}=0.1, \phi=0.0001, \alpha_{1}=0.1, \beta_{1}=0.1 \text {. }
$$

Substituting them into formula (3.2) gives

$$
\mu_{g}=0.0897 \% \text {. }
$$

Table 3 Results on risk for the AR (1)-GARCH $(1,1)$ process. The table reports the actual standard deviations of the simulated returns and their theoretical values for eight AR (1)-GARCH $(1,1)$ processes with different parameters. The actual standard deviation of the simulated returns is denoted by $\sigma_{\text {simul, }}$ and its theoretical value is denoted by $\sigma_{g}$

\begin{tabular}{lllllllll}
\hline Group & $(1)$ & $(2)$ & $(3)$ & $(4)$ & $(5)$ & $(6)$ & $(7)$ & $(8)$ \\
\hline$a_{1}$ & 0.1 & 0.1 & 0.1 & 0.1 & 0.1 & 0.1 & 0.1 & 0.1 \\
$\varphi$ & 0.0001 & 0.0001 & 0.0001 & 0.0001 & 0.0004 & 0.0004 & 0.0004 & 0.0004 \\
$a_{1}$ & 0.1 & 0.1 & 0.2 & 0.2 & 0.1 & 0.1 & 0.2 & 0.2 \\
$\beta_{1}$ & 0.1 & 0.2 & 0.1 & 0.2 & 0.1 & 0.2 & 0.1 & 0.2 \\
$\sigma_{g}$ & 0.0112 & 0.0120 & 0.0120 & 0.0129 & 0.0224 & 0.0239 & 0.0239 & 0.0259 \\
$\sigma_{\text {simul }}$ & 0.0114 & 0.0120 & 0.0121 & 0.0127 & 0.0224 & 0.0236 & 0.0238 & 0.0262 \\
\hline
\end{tabular}




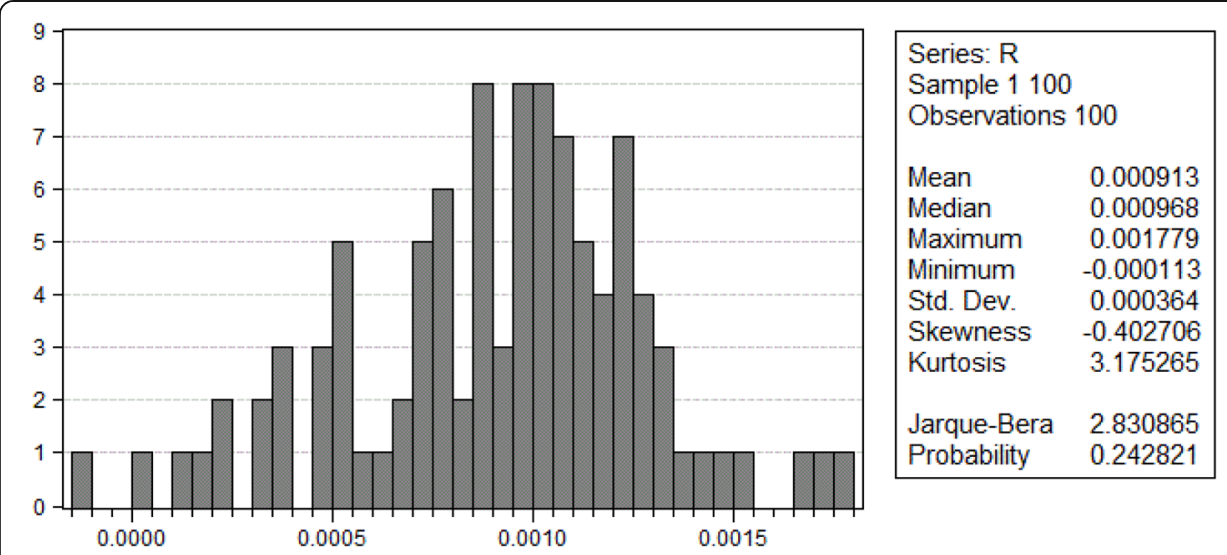

Fig. 5 The distribution and summary statistics of the average simulated return for the AR $(1)-G A R C H(1,1)$ process. We use $R$ to denote the average value of the simulated returns. The distribution of $R$ is shown in the left, and the summary statistics are listed in the right

Similarly, we simulated to produce a series consisting of 1000 observations according to eqs. (2.17), (2.40), and (2.41). Then, we simulated trading according to the strategy described in Definition 1 and generated a series of returns whose average value (denoted by $R$ ) can be computed and compared with the value from formula (3.2).

We carried out the simulation 100 times and found the mean of $R$ to be $0.0913 \%$, which approximates $0.0897 \%$.

Figure 5 shows the distribution of $R$ along with its summary statistics.

\section{Comparison with the method of conditional entropy}

In physics and information theory, Shannon entropy is used to measure the degree of disorder:

$$
S(i)=-\sum_{i=1}^{n} p(i) \log _{2} p(i)
$$

where $p(i)$ is the probability that the event $i$ appears out of $n$ events.

Based on this concept, Zhang proposed to measure market efficiency with conditional entropy (Wen et al. 2019):

$$
H(j)=-\sum_{i=1}^{n}\left[p(i) \sum_{j=1}^{m} p(j \mid i) \log _{2} p(j \mid i)\right],
$$

where $p(j \mid i)$ is the conditional probability that the event $j$ would follow, with the event $i$ given. Here, "event" stands for the direction of price movement. For example, $p(+\mid-$, $-,+)$ means the conditional probability that price will rise if the price history is "fall," "fall," and "rise" in turn for the recent three periods.

Conditional entropy uses the information from a given previous event (i.e., the past price) to help predict future price movement. The higher the conditional entropy is, the better the market efficiency is. For the binary case when $i$ and $j$ are both binary strings, its maximal value is one. Zhang (1999) did not present empirical research on the concept. 
Because the $\operatorname{MA}(\mathrm{q})$ process is equivalent to an $\operatorname{AR}(\infty)$ process, it is impossible to compute conditional entropy for the $\operatorname{MA}(q)$ or $\operatorname{ARMA}(p, q)$ process. Here, we just consider the AR (1) process.

First, given a set of parameters, we computed the expected value of the one-period excess return and the Sharpe ratio according to the corresponding formula.

Then, we simulated to produce a series consisting of 1000 observations according to the corresponding model.

For the AR (1) process, different absolute values of $a_{1}$ mean different degrees of market efficiency. The smaller the value of $\left|a_{1}\right|$ is, the better the market efficiency is. If $a_{1}$ is equal to zero, then the market is perfectly efficient, meaning the prices conform to a random walk.

Given $\sigma=0.15$, we simulated five times for each value of $a_{1}$ and computed conditional entropy according to formula (3.4).

Table 4 displays the results for nine sets of parameters.

The first column in Table 4 is the result for random walk. As expected, its Sharpe ratio is equal to zero, and its conditional entropy is very close to one.

The SR in the second column is equal to 0.0359 , but the conditional entropy is nearly the same as the one in the first column. It shows that the conditional entropy is not a proper measure of market efficiency when the signal-noise ratio of the market is at a low level.

Figure 6 shows the scatter plot corresponding to Table 4.

From Fig. 6 and Table 4, we can observe that the conditional entropy declines when the SR rises to a value larger than 0.1 though its range of fluctuation expands correspondingly. In summary, both the Sharpe ratio and conditional entropy can be used to measure market efficiency. However, the former is more accurate than the latter, especially when the degree of market efficiency is very high.

\section{Empirical research}

As examples, we measured the market efficiency of the S\&P 500 Index and the SSE Composite Index. The two samples span from January 1, 2000 to December 31, 2014. From Yahoo finance, we obtained a total of 782 weekly closing prices of the S\&P 500 Index, and a total of 754 weekly closing prices of the SSE Composite Index. Returns were the log differences of the closing prices.

Table 4 Results on conditional entropy. The table reports the Sharpe ratio and conditional entropy for nine AR (1) processes with different parameters. The Sharpe ratio is denoted by $S R$, and the conditional entropy is denoted by $H$

\begin{tabular}{|c|c|c|c|c|c|c|c|c|c|}
\hline Group & (1) & (2) & (3) & (4) & (5) & (6) & (7) & (8) & (9) \\
\hline$a_{1}$ & 0 & 0.045 & 0.145 & 0.245 & 0.345 & 0.445 & 0.545 & 0.645 & 0.745 \\
\hline$\mu_{g}(\%)$ & 0 & 0.0539 & 0.1754 & 0.3024 & 0.4399 & 0.5947 & 0.7780 & 1.0102 & 1.3367 \\
\hline$S R$ & 0 & 0.0359 & 0.1165 & 0.1993 & 0.2863 & 0.3798 & 0.4829 & 0.6002 & 0.7392 \\
\hline \multirow[t]{5}{*}{$H$} & 0.9960 & 0.9985 & 0.9968 & 0.9835 & 0.9706 & 0.9453 & 0.9018 & 0.8461 & 0.7835 \\
\hline & 0.9995 & 0.9966 & 0.9897 & 0.9869 & 0.9597 & 0.8900 & 0.8958 & 0.8571 & 0.7892 \\
\hline & 0.9994 & 0.9978 & 0.9923 & 0.9823 & 0.9546 & 0.9305 & 0.8800 & 0.8704 & 0.7984 \\
\hline & 0.9978 & 0.9933 & 0.9919 & 0.9724 & 0.9180 & 0.9493 & 0.9101 & 0.8694 & 0.7760 \\
\hline & 0.9979 & 0.9979 & 0.9875 & 0.9773 & 0.9578 & 0.9379 & 0.9138 & 0.8466 & 0.7887 \\
\hline
\end{tabular}




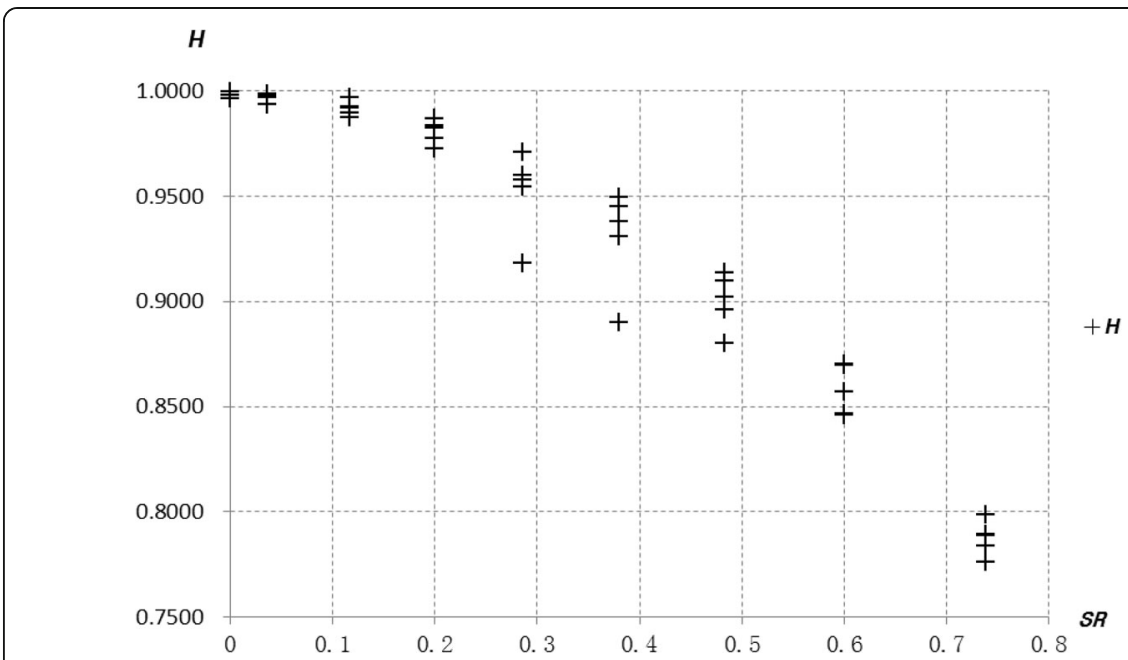

Fig. 6 The relationship between the Sharpe ratio and conditional entropy. The horizontal axis shows the Sharpe ratio, and the vertical axis shows the conditional entropy

We chose the $\operatorname{AR}(p)$-GARCH $(1,1)$ process to apply to the data. Table 5 reports the parameter estimates for the time series model.

Table 5 shows that the ARCH and GARCH effects are always significant at the $1 \%$ level in the two models. We can compute the R-squared according to the residual error and the data of returns. Then, we can obtain the Sharpe ratio according to formula (2.16). For the S\&P 500 Index, the Sharpe ratio is 0.055; for the SSE Composite Index, the Sharpe ratio is 0.086 . So, we conclude that the market efficiency of the S\&P 500 Index is higher than that of the SSE Composite Index.

Based on the AR-GARCH model, we propose a trading strategy on the S\&P 500 Index, which we call the AR-GARCH strategy. We compare the return of the strategy

Table 5 Parameter estimates for the AR(p)-GARCH $(1,1)$ process. The table reports the parameter estimates for the AR(p)-GARCH $(1,1)$ processes. The notations come from expressions (2.2), (2.40), and (2.41). The numbers in parentheses are the $t$-ratios. The $t$-ratios marked with asterisks (double asterisks or three asterisks) indicate that the corresponding coefficients are statistically different from zero at the $10 \%$ ( $5 \%$ or $1 \%$ ) level of significance

\begin{tabular}{lll}
\hline & S\&P 500 Index & SSE Composite Index \\
\hline$a_{1}$ & -0.0782383 & 0.0511606 \\
& $(-1.87)^{*}$ & $(1.38)$ \\
$a_{2}$ & -0.0082874 & 0.0837641 \\
& $(-0.20)$ & $(2.17)^{* *}$ \\
$a_{3}$ & -0.0786243 & - \\
& $(-2.16)^{* *}$ & \\
Intercept & 0.0025749 & 0.0006806 \\
& $(3.72)^{* * *}$ & $(0.65)$ \\
$a_{1}$ & 0.7341439 & 0.8754173 \\
& $(23.76)^{* * *}$ & $(28.85)^{* * *}$ \\
$\beta_{1}$ & 0.2185006 & 0.0910423 \\
& $(8.59)^{* * *}$ & $(4.17)^{* * *}$ \\
$\varphi$ & 0.0000358 & 0.0000367 \\
Log Likelihood & $(3.92)^{* * *}$ & $(2.63)^{* * *}$ \\
\hline & 1867.151 & 1542.614 \\
\hline
\end{tabular}


to the buy-and-hold strategy over the sample period. The merit of the strategy is captured in Definition 1, as also mentioned in "The Sharpe Ratio based on the ARMAGARCH Forecast" section. It is namely that if the prediction of the AR-GARCH model is positive, we should buy the index at the closing price. Conversely, we should sell the index at the closing price if the prediction is negative. We carried out the strategy on a rolling basis without considering trading friction.

Firstly, for each trading week, we used the previous 50 weekly yields of the S\&P 500 Index as a sample window to fit the optimal AR-GARCH model. Secondly, we obtained the predicted yield of the next trading week through the estimated model. Thirdly, if the prediction of the next week was positive, then we went long the index at the latest closing price; if the prediction was negative, we went short the index at the same price. Lastly, for the next trading week, in case we got the same direction of the prediction as the previous week, then we held our position. If not, then we closed our position and made a reverse position at the close price. Based on the strategy, we got the following two curves in the Fig. 7.

The AR-GARCH strategy did not always outperform the buy-and-hold strategy. The excess return of the AR-GARCH strategy most occurred during the financial crisis period of 2008 and in the period of the tech bubble burst of 2001. While in other period, the AR-GARCH strategy could not gain excess return obviously better than the buy-and-hold strategy.. This may mean that during these crisis periods, the market was not as effective as the ordinary period. This may also mean that one can obtain excess returns through the time series strategy only when the market is not efficient. During these periods, the model predicts better because of the available past information.

To show the results' stability, we fit the daily and monthly data to the AR-GARCH process. Table 6 reports the parameter estimates for the four series.

Table 6 shows that the ARCH and GARCH effects are always significant at the 1\% level in all four models. Formula (2.16) was used to compute the Sharp ratios for the S\&P 500 Index, which were 0.0745 and 0.0515 for the monthly and daily data,

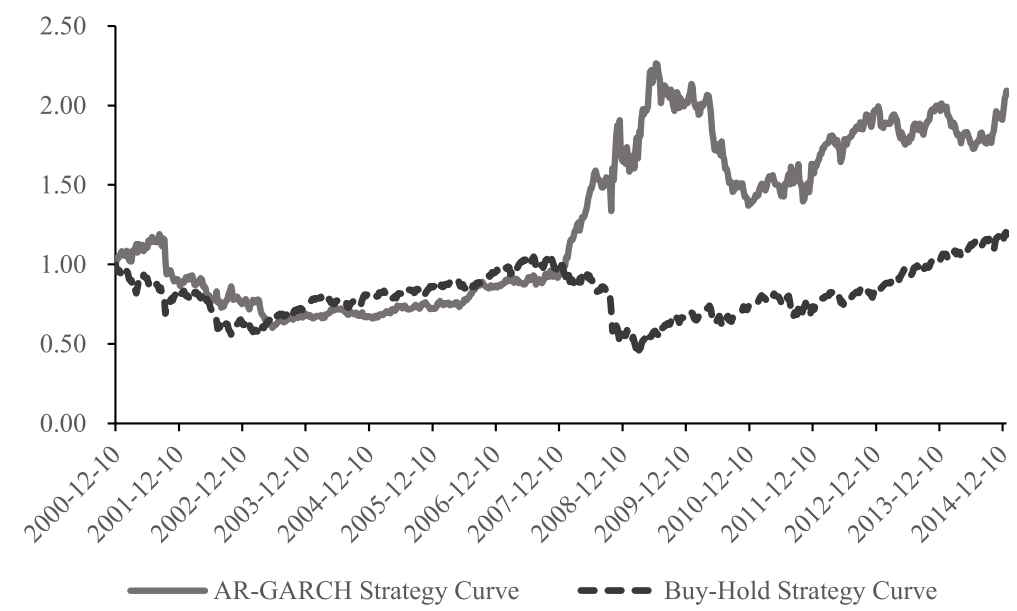

Fig. 7 The AR-GARCH strategy curve and the buy-and-hold strategy curve. The AR-GARCH strategy is not always effective. Its returns mainly occurred in the crisis period of 2008. During other periods, the strategy did not perform better than the buy-and-hold strategy. This means that the market is more efficient during ordinary times than during the crisis period. In the latter period, one can get excess returns in the inefficient market 
Table 6 Parameter estimates for the AR-GARCH process. The table reports the parameter estimates for the $\operatorname{AR}(p)-G A R C H(1,1)$ processes for daily and $\operatorname{AR}(p)-G A R C H(1,0)$ processes for monthly data. The notations come from expressions (2.2), (2.40) and (2.41). The numbers in parentheses are the $t$ ratios. The t-ratios marked with asterisks (double asterisks or three asterisks) indicate that the corresponding coefficients are statistically different from zero at 10\% (5\% or $1 \%$ ) level of significance

\begin{tabular}{lllll}
\hline & $\begin{array}{l}\text { S\&P 500 Index } \\
\text {-monthly }\end{array}$ & $\begin{array}{l}\text { SSE Composite } \\
\text { Index-monthly }\end{array}$ & $\begin{array}{l}\text { S\&P 500 Index } \\
\text {-daily }\end{array}$ & $\begin{array}{l}\text { SSE Composite } \\
\text { Index-daily }\end{array}$ \\
\hline$a_{1}$ & 0.151447 & 0.080948 & -0.058303 & 0.018532 \\
& $(2.54)^{* *}$ & $(1.11)$ & $(-3.13)^{* * *}$ & $(1.10)$ \\
$a_{2}$ & & 0.193746 & -0.032937 & -0.011153 \\
& & $(2.81)^{* * *}$ & $(-1.94)^{*}$ & $(-0.67)$ \\
$a_{3}$ & - & & 0.022778 \\
Intercept & & & & $(1.36)$ \\
& 0.002195 & 0.003046 & 0.000583 & 0.000335 \\
$a_{1}$ & $(0.60)$ & $(0.54)$ & $(4.04)^{* * *}$ & $(1.73)$ \\
$\beta_{1}$ & 0.960079 & 0.973209 & 0.895647 & 0.913373 \\
& $(18.08)^{* * *}$ & $(78.52)^{* * *}$ & $(111.8)^{* * *}$ & $(160.7)^{* * *}$ \\
$\varphi$ & & & 0.092683 & 0.075154 \\
Log Likelihood & 306.2535 & & $(12.64)^{* * *}$ & $(14.11)^{* * *}$ \\
\hline
\end{tabular}

respectively. The Sharp ratios of the SSE Composite Index were 0.122 and 0.056 for the monthly and daily data, respectively. So, we can also conclude that the market efficiency of the S\&P 500 Index is higher than that of the SSE Composite Index.

\section{Conclusion}

From the empirical research perspective, it is more meaningful to measure relative efficiency of markets as opposed to testing the EMH. A proper measure of market efficiency can reflect the potential return and the relevant risk. However, the traditional test statistics reflect neither of these two aspects. In this paper, we proposed a new method for comparing the weak-form efficiency of a market. We deduced the formulae of the expected return and risk from the ARMA-GARCH model and thereby acquired the formula of the Sharpe ratio. The study found that the Sharpe ratio is not affected by the variance of $\varepsilon_{t}$ and thus not affected by the GARCH process. The Sharpe ratio just depends on the coefficients of the ARMA $(p, q)$ process, namely $a_{1}, \cdots, a_{p}, b_{1}, \cdots, b_{q}$.

In empirical research, the Sharpe ratio can be formulated with a monotonic increasing function of $R$-squared if the sample size is large enough. This shows that the $R$-squared in time series models is of great significance for the measure of market efficiency. One can utilize the Sharpe ratio to measure weak-form efficiency and compare different markets. The results of our stochastic simulation demonstrated the validity of the proposed method. It can be applied in both high and low efficiency markets.

\section{Appendix: Proofs}

Lemma 1: Provided that $\left\{Y_{t}\right\}$ satisfies the $\operatorname{ARMA}(p, q)$ process, and both the risk-free return and the trading cost rate are zero, trading in accordance with the strategy described in Definition 1 will produce a series of excess returns. 
If the standard deviation of $E_{t}\left[Y_{t+1}\right]$ is known and denoted by $\sigma_{E}$, then the expected value of one-period excess return is

$$
\mu_{g}=\frac{\sigma_{E} \sqrt{2}}{\sqrt{\pi}} .
$$

Meanwhile, the standard deviation of one-period excess return is

$$
\sigma_{g}=\left[\sigma^{2}+\left(1-\frac{2}{\pi}\right) \sigma_{E}^{2}\right]^{\frac{1}{2}} .
$$

Proof of Lemma 1: According to the original assumption, we know

$$
E_{t}\left[Y_{t+1}\right] \sim N\left(0, \sigma_{E}\right) .
$$

According to eq. (2.8), we have

$$
\begin{aligned}
\mu_{g} & =E\left\{Y_{t+1} \mid E_{t}\left(Y_{t+1}\right)>0\right\} \\
& =E\left\{E_{t}\left(Y_{t+1}\right) \mid E_{t}\left(Y_{t+1}\right)>0\right\} \\
& =\int_{0}^{+\infty} \frac{2 x}{\sqrt{2 \pi} \sigma_{E}} e^{-\frac{x^{2}}{2 \sigma_{E}^{2}}} d x \\
& =\frac{\sigma_{E} \sqrt{2}}{\sqrt{\pi}} \int_{0}^{+\infty} e^{-\frac{x^{2}}{2 \sigma_{E}^{2}}} d\left(\frac{x^{2}}{2 \sigma_{E}^{2}}\right) \\
& =\frac{\sigma_{E} \sqrt{2}}{\sqrt{\pi}} * e^{-\frac{x^{2}}{2 \sigma_{E}^{2}}} \mid 0 \\
& =\frac{\sigma_{E} \sqrt{2}}{\sqrt{\pi}}
\end{aligned}
$$

This is just formula (2.9).

Because $\varepsilon_{t+1}$ and $E_{t}\left[Y_{t+1}\right]$ are uncorrelated, according to eq. (2.5) and Definition 1, we have

$$
\begin{aligned}
\sigma_{g}^{2} & =\sigma^{2}+\operatorname{Var}\left\{E_{t}\left(Y_{t+1}\right) \mid E_{t}\left(Y_{t+1}\right)>0\right\} \\
& =\sigma^{2}+E\left\{\left[E_{t}\left(Y_{t+1}\right)\right]^{2} \mid E_{t}\left(Y_{t+1}\right)>0\right\}-E\left\{\left[E\left[E_{t}\left(Y_{t+1}\right) \mid E_{t}\left(Y_{t+1}\right)>0\right]\right]^{2}\right\} .
\end{aligned}
$$

Substituting expression (A.1) into eq. (A.2) gives

$$
\begin{aligned}
\sigma_{g}^{2} & =\sigma^{2}+E\left\{Y_{t+1}^{2} \mid E_{t}\left[Y_{t+1}\right]>0\right\}-\frac{2 \sigma_{E}^{2}}{\pi} \\
& =\sigma^{2}-\frac{2 \sigma_{E}^{2}}{\pi}+\int_{0}^{+\infty} \frac{2 x^{2}}{\sqrt{2 \pi} \sigma_{E}} e^{-\frac{x^{2}}{2 \sigma_{E}^{2}}} d x \\
& =\sigma^{2}-\frac{2 \sigma_{E}^{2}}{\pi}+\int_{-\infty}^{+\infty} \frac{x^{2}}{\sqrt{2 \pi} \sigma_{E}} e^{-\frac{x^{2}}{2 \sigma_{E}^{2}}} d x \\
& =\sigma^{2}-\frac{2 \sigma_{E}^{2}}{\pi}+\sigma_{E}^{2} \\
& =\sigma^{2}+\left(1-\frac{2}{\pi}\right) \sigma_{E}^{2} .
\end{aligned}
$$

This is just formula (2.11).

\section{Authors' contributions}

Lin Liu contributed the central idea, conceived and designed this research, supervised the study, provided mathematical and model Derivation, and wrote the initial draft of the paper. Qiguang Chen contributed to refining the ideas, performed the data and statistical analysis, carrying out additional analyses and coordination and finalizing this manuscript. Both authors wrote, corrected and agreed to the published version of the manuscript. Both authors have read and agreed to the published version of the manuscript. 
Funding

This research received no external funding.

\section{Availability of data and materials}

The datasets used or analyzed during the current study are available from the authors on reasonable request.

\section{Competing interests}

The authors declare that they have no competing interests.

Received: 29 November 2019 Accepted: 29 August 2020

Published online: 15 October 2020

\section{References}

Box G, Jenkins GM, Reinsel GC (2008) Time series analysis. Wiley, NJ

Box G, Pierce D (1970) Distribution of residual autocorrelations in autoregressive-integrated moving average time series models. J Am Stat Assoc 65:1509-1526

Campbell JY, Lo AW, Mackinlay AC (1997) The econometrics of financial markets. Princeton University Press, Princeton Cochrane JH (1999) Portfolio advice for a multifactor world. Econ Perspect 23:59-78

Fama EF (1970) Efficient capital markets: a review of theory and empirical work. J Financ 25:383-417

Fang Y, Xu D (2003) The predictability of asset returns: an approach combining technical analysis and time series forecasts. Int J Forecast 19:369-385

Griffin JM, Kelly PJ, Nardari F (2010) Do market efficiency measures yield correct inferences? A Comparison of Developed and Emerging Markets. Rev Financ Stud 23:3225-3277

Hamilton JD (1994) Time series analysis. Princeton University Press, Princeton

Han CY, Wang YM, Ning Y (2019) Analysis and comparison of the Multifractality and efficiency of Chinese stock market: evidence from dynamics of major indexes in different boards. Phys A 528:121305

Hou K, Moskowitz TJ (2005) Market frictions, Price delay, and the cross-section of expected returns. Rev Financ Stud 18:9811020

Huang D, Zhou GF (2017) Upper bounds on return predictability. J Financ Quant Anal 52:401-425

Kirby C (1998) The restrictions on predictability implied by rational asset pricing models. Rev Financ Stud 11:343-382

Levich RM, Poti V (2015) Predictability and 'good deals' in currency markets. Int J Forecast 31:454-472

Liu J, Cheng C, Yang XL, Yan LZ, Lai YZ (1999) Analysis of the efficiency of Hong Kong REITs market based on Hurst exponent. Phys A 534:122035

Lo AW, Mackinlay AC (1988) Stock market prices do not follow random walks: evidence from a simple specification test. Rev Financ Stud 1:41-66

Lu M (1999) Advanced time series econometrics. Shanghai People's Publishing House, Shanghai

Malkiel B (1992) Efficient market hypothesis. In: Newman P, Milgate M, Eatwell J (eds) New Palgrave dictionary of money and finance. London, Macmillan

Mech T (1993) Portfolio return autocorrelation. J Financ Econ 34:307-344

Moradi M, Nooghabi MJ, Rounaghi MM (2019) Investigation of Fractal Market Hypothesis and Forecasting Time Series Stock Returns for Tehran Stock Exchange and London Stock Exchange. Int J Financ Econ 11:1002/IJFE.1809

Ola MR, Nooghabi MJ, Rounaghi MM (2014) Chaos process testing (using local polynomial approximation model) in predicting stock returns in Tehran stock exchange. Asian J Res Bank Financ 4:100-109

Poti V (2018) A new tight and general bound on return predictability. Econ Lett 162:140-145

Ross SA (2005) Neoclassical Finance. Princeton: Princton University Press

Sharpe WF (1966) Mutual Fund Performance. J Bus 39:119-138

Wen FH, Xu LH, Ouyang GD, Kou G (2019) Retail investor attention and stock Price crash risk: evidence from China. Int Rev Financ Anal 65:101376

Zhang Y (1999) Toward a theory of marginally efficient markets. Phys A 269:30-40

\section{Publisher's Note}

Springer Nature remains neutral with regard to jurisdictional claims in published maps and institutional affiliations.

\section{Submit your manuscript to a SpringerOpen ${ }^{\circ}$ journal and benefit from:}

- Convenient online submission

- Rigorous peer review

- Open access: articles freely available online

High visibility within the field

- Retaining the copyright to your article

Submit your next manuscript at $\boldsymbol{\nabla}$ springeropen.com 